\title{
Information processing in preschool children
}

\author{
John F. Santa Larbara Jr. and William P. Paré
}

BOSTON COLLEGE

\begin{abstract}
Preschool children between the ages of 3 and 5 were asked to tell $\mathrm{E}$ whether the two figures on a stimulus card were "exactly the same, or different." The stimulus cards were classified into seven levels of complexity, based upon the number of "bits"'each figure contained. It was found that the 3,4 , and 5-yr. old Ss performed better than chance at successively higher levels of complexity.
\end{abstract}

\section{Problem}

Miller (1956) has reviewed several studies concerned with the amount of information, measured in "bits," which can be processed by various sense modalities. A "bit" of information is that amount of information required to reduce uncertainty by one-half. It is simply a means of quantifying stimulus complexity. A logarithmic relationship exists between stimulus complexity and the number of bits contained in that stimulus. It may be expressed as $\mathrm{H}=\log _{2} \mathrm{n}$, where $\mathrm{H}$ is the number of bits, and $n$ is the number of units in the stimulus.

If this sensory ability to process information develops with age, its measurement in young children would be valuable to developmental theorists who emphasize the relation of perceptual development to cognitive growth (Piaget, 1947; Hebb, 1949; Brunner, 1963). The advantage in using the concept of bits lies in the fact that various sensory modalities may be compared in equivalent terms. It also allows perceptual abilities to be compared with cognitive functions on the same basis. There is also a difficulty involved in using the concept of bits. It centers on the issue of defining which stimulus elements are dominating S's attention. This difficulty has been met in the present study by using the number of arms or prongs in the stimulus figures (cf. Fig. 1). Granted that the elements which $\mathrm{S}$ may attend to are varied (e. g., number of lines or angles, figure density, etc.), all of these possible cues are directly related to the number of prongs in the figure. The present study is an attempt to use the measure of bits in determining the ability of preschool children to make identity-nonidentity judgements.

\section{Method}

Ss were children age $3(\mathrm{~N}=12), 4 \quad(\mathrm{~N}=15)$, and $5(\mathrm{~N}=12)$, who attended the Campion Children's Class at Boston College. Each $\mathrm{S}$ was presented with 28 stimulus cards. Each card contained two figures which were either identical or different only in terms of the number of prongs in each. The patterns in Fig. 1 were repeated throughout all seven levels of complexity. There were four cards at each level; Three nonidentical pairs and one identical pair. At the first level of complexity, the nonidentical pairs
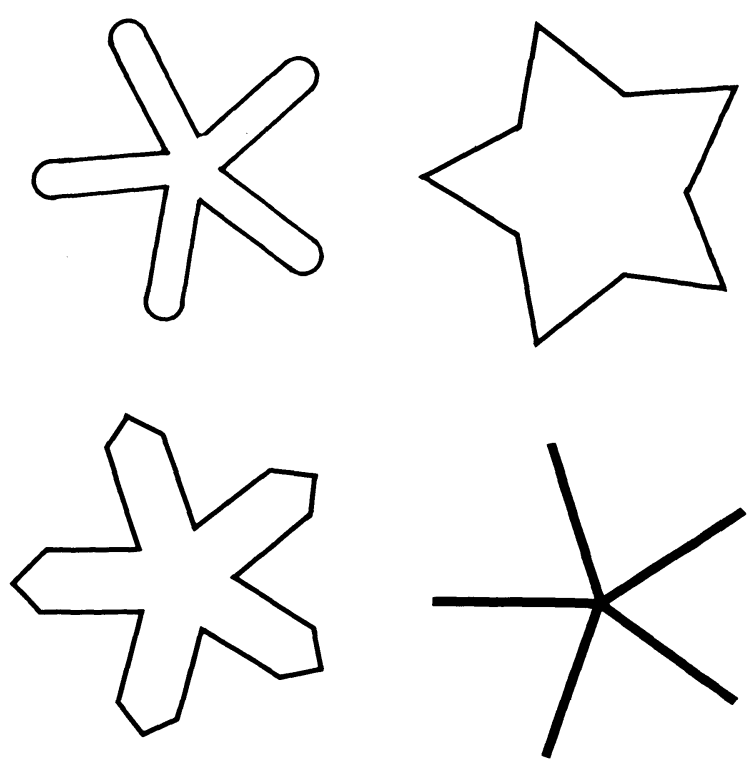

Fig. 1. Sample of stimulus figures.

contained five and seven prongs, and the identical pairs contained six prongs each. As complexity increased from one level to another, one prong was added to each figure. A constant difference of two prongs was thus maintained throughout all levels of complexity. The range, in terms of number of prongs, was from 5,7 to 11,13 . The transformation into bits was made from Attneave's table (1959, p. 100). The range of bits was from $2.32,2.81$ to $3.46,3.70$. Table 1 indicates the complexity, expressed in both bits and units, of the figures at each level.

The stimulus cards were arranged in order of ascending complexity, the identical cards being randomly scattered among the nonidentical cards. The order was held constant for all Ss. The nonidentical cards were flipped randomly so that the more complex of the two was not always on the same side. The cards were presented one at a time and $\mathrm{S}$ was allowed to hold the cards. S was asked to tell $\mathrm{E}$ if the two figures were "exactly the same, or different." A brief pretest period was spent familiarizing $\mathrm{S}$ with the precedure.

\section{Results}

Figure 2 illustrates the large differences between age groups at different levels of complexity. An analysis of variance indicated that these differences were significant for the main effects of both age $(F=$ 16.37; $\mathrm{df}=3 / 36 ; \mathrm{p}<.001$ ) and level of complexity 
$(F=4.07 ; d f=6 / 216 ; p<.001)$. Figure $z$ indicates that the $3-y r$. old group could not distinguish between identical and nonidentical figures beyond the second level of complexity. The 4-yr. old group faltered after the third level was reached. The 5-yr. old group showed a much greater ability, performing well above chance at all but the sixth level of complexity.

The mean of response latencies (not presented here) showed that the only instances of increased latency occurred in the 5-yr. old group at the fifth, sixth, and seventh levels of complexity.

Separate analysis of nonidentical figures only, indicated that performance was slightly poorer on these figures for all age groups.

\section{Discussion}

The results indicate that 3 and 4-yr. old children cannot 'process' visual information as well as adults, if Miller's (1956) review is indicative of adult performance. Although the 5-yr. old group did better than chance, they did make more errors than the few adults tested in a separate study. This ability to process information then does develop with age, and none of the age groups studied reached a level of adult performance.

Such a measure of perceptual ability as presented here would also be valuable for other sensory modalities.

Table 1. Description of Stimulus Pairs

in Terms of Elements and Bits

Level of Description of Complexity

Complexity Elements* Bits*

$\begin{array}{lll}1 & 5,7 & 2.32,2.81 \\ 2 & 6,8 & 2.58,3.00 \\ 3 & 7,9 & 2.81,3.17 \\ 4 & 8.10 & 3.00,3.32 \\ 5 & 9,11 & 3.17,3.46 \\ 6 & 10,12 & 3.32,3.58 \\ 7 & 11,13 & 3.46,3.70\end{array}$

* Two descriptive numbers are presented

since each stimulus card contains two

figures.

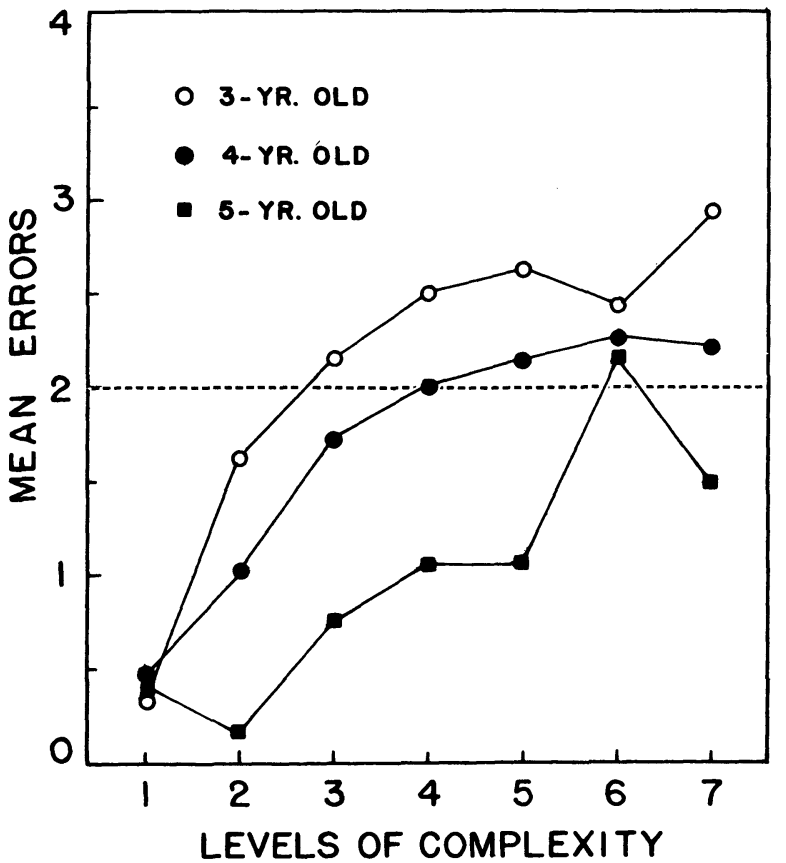

Fig. 2. Mean errors per group at each level of complexity.

First of all, such measures could provide operational definitions for such concepts as Bruner's (1963) "iconic representation." Secondly, the measures would be valuable in determining the limits imposed on cognitive growth by perceptual development. Whether these perceptual limits are themselves determined by cognitive abilities, or by the capacity of the maturing sensory organ, is an open question. Logitudinal studies using the measure of bits could also offer information as to when symbolic manipulation comes to dominate a simple sensory ability. The hypothesis being that a great deal more information would be processed with symbolic dominance.

\section{References}

ATTNEAVE, F. Application of information theory to psychology: A summary of basic concepts, methods, and results. New York: Henry Holt, 1959.

BRUNER, J. S. On intellectual growth. Paper presented at the American Psychological Association Meeting, Philadelphia, 1963. HEBB, D. O. Organization of behavior. New York: Wiley, 1949. MILLER, G. A. The magic number seven, plus or minus two: Some limits on our capacity for processing information. Psychol. Rev., 1956, 63, 81-97.

PIAGET, J. The psychology of intelligence. Paterson, N. J.: Littlefield, Adams \& Co., 1960. 